\title{
Obesity as a Risk Factor for Diabetes Mellitus in the Local Population of Pakistan
}

\author{
Samreen Riaz \\ Department of Microbiology and Molecular Genetics, University of the Punjab, Lahore, Pakistan \\ *Corresponding Author: samreen.mmg@pu.edu.pk
}

Copyright $(2014$ Horizon Research Publishing All rights reserved.

\begin{abstract}
A worldwide epidemic exists with respect to diabetes mellitus because of increased rates of obesity. There is a significant correlation between obesity and insulin resistance and obesity causes the increase in the severity of the disease. The adipose tissues in the visceral region function as an endocrine organ that produces certain proteins with role in glucose homeostasis. The expression level of some of these proteins is increased in diabetes and can serve as specific marker of the disease. The study was performed to check the effect of various physical and biochemical parameters of obesity on the development and progression of diabetes mellitus. In our study the serum samples of diabetics were collected and were further categorized into two groups i.e. diabetic obese and diabetic non obese, on the basis of BMI, waist circumference and waist to hip ratio. The fasting blood sugar level, the total protein content, along with the complete lipid profile was performed. A marked increase in the total protein content and lipid profile was observed in diabetic obese as compared to diabetic non obese and control group. The results were found to be significant with $p<0.05$. The lipid profile of diabetic obese was characterized by high cholesterol, LDL and VLDL concentration and low HDL concentration while that of control was characterizes by high HDL level and low cholesterol, LDL and VLDL level. The adipose secreted proteins whose expression is thought to increase in diabetes are studied. For this purpose the protein profile of serum was obtained on 10\% SDS PAGE and the gels were stained with coomassie R-250 and silver salts. The proteins with exacerbated expression in diabetic obese individuals were studied. The expression of proteins such as leptin (16000 dalton), C reactive protein (25039 dalton), TNF alpha (25644 daltons), apolipoprotein E (34000 dalton), alpha 2 macroglobulin (163278 dalton) and apolipoprotein B (250000 dalton) was observed to be increased in diabetic obese as compared to diabetic non obese and control. There inflammation proteins secreted by adipose tissues cause the increase in the severity and progression of diabetes mellitus in the population.
\end{abstract}

Keywords Obesity, Diabetes, Pakistani Population

\section{Introduction}

Diabetes mellitus is a world health problem and affects all human society at various stages of development. It is more common amongst developed countries where affluent and over weight individual lives longer than human being of under developed countries. The incidence of this disease in a society, whether in Pakistan or any developed country is difficult to judger but it is quiet obvious that the disease is multiplying geometrically more or less, because of genetic and environmental factors [1].

Diabetes mellitus is a group of metabolic diseases characterized by hyperglycemia resulting from defects in insulin secretion, insulin action, or both. The chronic hyperglycemia of diabetes is associated with long-term damage, dysfunction, and failure of various organs, especially the eyes, kidneys, nerves, heart, and blood vessels. Several pathogenic processes are involved in the development of diabetes. These range from autoimmune destruction of the $\beta$-cells of the pancreas with consequent insulin deficiency to abnormalities that result in resistance to insulin action. The basis of the abnormalities in carbohydrate, fat, and protein metabolism in diabetes is deficient action of insulin on target tissues. Type 2 diabetes is most common form of the diabetes accounting for $90 \%$ of cases [2].

\subsection{Regulation of Blood Glucose Levels}

The maintenance of the blood sugar at normal levels is brought about by an efficient regulatory mechanism. The main organs in this mechanism are the liver, the autonomic nervous system, and certain glands of internal secretion called endocrine glands. Maintenance of blood glucose homeostasis is of paramount importance to the survival of the human organism. The predominant tissue responding to signals that indicate reduced or elevated blood glucose levels is the liver. Indeed, one of the most important functions of the liver is to produce glucose for the circulation. Both elevated and reduced levels of blood glucose trigger hormonal responses to initiate pathways designed to restore glucose homeostasis. Low blood glucose triggers release of 
glucagon from pancreatic beta-cells. High blood glucose triggers release of insulin from pancreatic beta-cells.

Diabetes results from the dysregulation of multiple glucoregulatory hormones that normally act to maintain glucose homeostasis. These hormonal imbalances lead to chronic hyperglycemia, which results in an array of microvascular complications including retinopathy, nephropathy, and neuropathy. Any defect in insulin production leads to improper regulation of glucose in the blood and result in diabetes. Similarly, in patients with type 1 and type 2 diabetes, postprandial glucagon secretion is abnormally elevated. This inappropriate secretion of glucagon leads to excess hepatic glucose production and is an important contributor to postprandial hyperglycemia in patients with diabetes as in figure 1 [3].

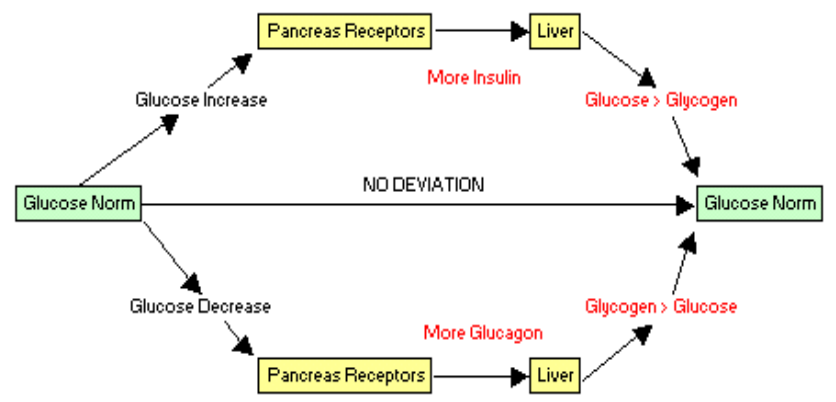

Figure 1. Glucose Homeostasis

\section{What is Insulin?}

Insulin is a peptide hormone secreted by the $\beta$ cells of the pancreatic islets of Langerhans and maintains normal blood glucose levels by facilitating cellular glucose uptake, regulating carbohydrate, lipid and protein metabolism and promoting cell division and growth through its mitogenic effects. It is synthesized in the $\beta$ cells of the pancreatic islets of langherhans as its precursor, proinsulin. Glucose is the principal stimulus for insulin secretion, though other macronutrients, hormones, humoral factors and neural input may modify this response. Insulin, together with its principal counter-regulatory hormone glucagon, regulates blood glucose concentrations. Pancreatic $\beta$ cells secrete $0.25-1.5$ units of insulin per hour during the fasting (or basal) state, sufficient to enable glucose insulin-dependent entry into cells. [4].

\subsection{Insulin Resistance}

Insulin is released by the pancreas in response to the presence of sugar (glucose) in the blood stream. Glucose gets into the blood through consumption of sugar or sugar-containing foods or via the breakdown of carbohydrates into simple sugars. Insulin is a chemical messenger that acts on the walls of the cells to cause the release, from within the cell, of special protein molecules - the so-called GLUT-4 transporters. The GLUT-4 transporters rise to the cells' outer membranes where they grab hold of the glucose molecule and transport it into the interior of the cell. Here the glucose is used to produce energy and any excess is converted into fat Insulin resistance is defined where a normal or elevated insulin level produces an attenuated biological response; classically this refers to impaired sensitivity to insulin mediated glucose disposal Insulin resistance in most cases is believed to be manifest at the cellular level via post-receptor defects in insulin signalling. Possible mechanisms include down-regulation, deficiencies or genetic polymorphisms of tyrosine phosphorylation of the insulin receptor, IRS (insulin receptor substrate) proteins or may involve abnormalities of GLUT 4 function ( Figure 2).

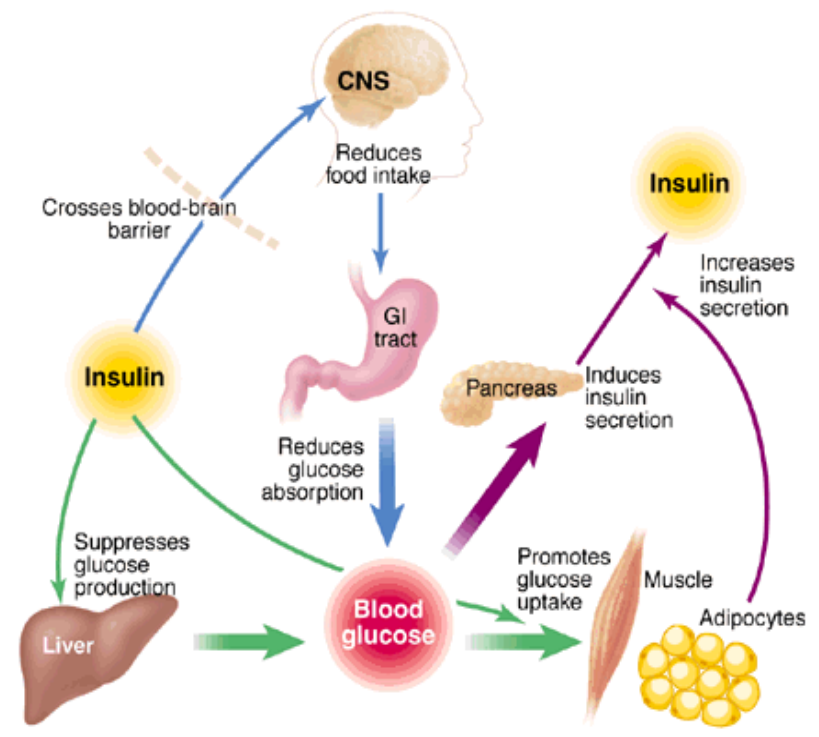

Figure 2. Mode Of Action Of Insulin And Normal Regulation Of Blood Glucose Levels.

\section{Types of Diabetes}

There are several different types of diabetes, varying very slightly from one another. Different types are classified as follows [5]

\subsection{Type 1 Diabetes}

This form of diabetes, which accounts for only $5-10 \%$ of those with diabetes, previously encompassed by the terms insulin-dependent diabetes, type I diabetes, or juvenile-onset diabetes, results from a cellular-mediated autoimmune destruction of the $\beta$-cells of the pancreas. Autoimmune destruction of $\beta$-cells has multiple genetic predispositions and is also related to environmental factors that are still poorly defined. Although patients are rarely obese when they present with this type of diabetes, the presence of obesity is not incompatible with the diagnosis. [6-7]. 


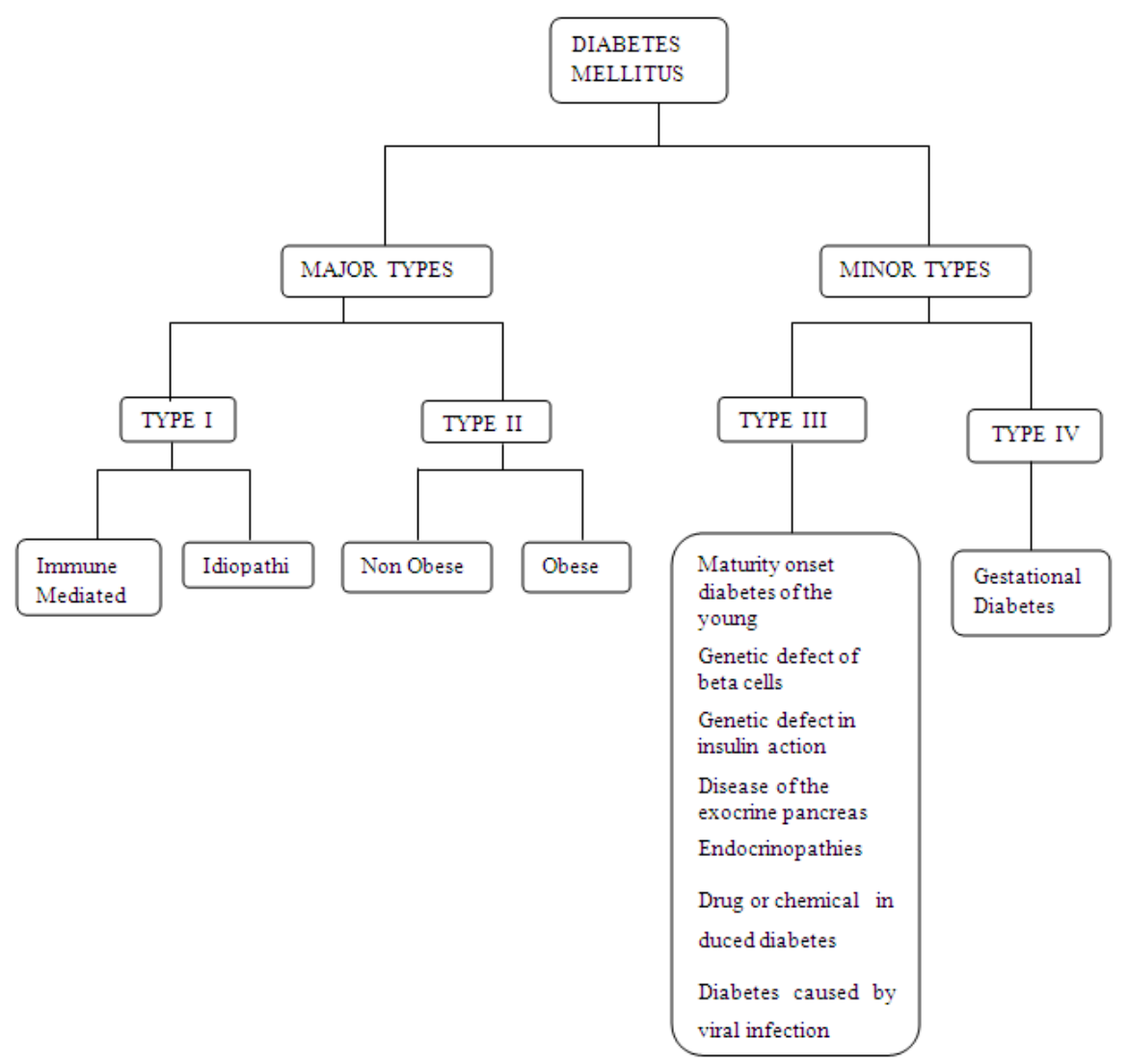

Figure 3. Classification of the major types of Diabetes mellitus.

\subsection{Type 2 Diabetes}

This form of diabetes, which accounts for $\sim 90-95 \%$ of those with diabetes, previously referred to as non-insulin-dependent diabetes, type II diabetes, or adult-onset diabetes, encompasses individuals who have insulin resistance and usually have relative (rather than absolute) insulin deficiency. Most patients with this form of diabetes are obese, and obesity itself causes some degree of insulin resistance. Patients who are not obese by traditional weight criteria may have an increased percentage of body fat distributed predominantly in the abdominal region [21]. See Figure 3.

\section{What is Obesity?}

Obesity represents an increase in adipose tissue mass or it specifically refers to an excess amount of body fat. Every individual needs a certain amount of body fat for energy, heat insulation and shock absorption. However, excessive deposition of fat in the body, which is usually referred to as overweight or obesity, is dangerous. Overweight specifically refers to an excess body weight compared to set standards, while obesity is to have an abnormally high proportion of total body fat. Obesity is a state of low-grade chronic inflammation, as indicated by the increased concentrations of C-reactive protein, IL-6, and other inflammatory markers identified in the plasma of obese individuals [8].
Obesity is a major health hazard all over the world and is becoming a major health threat in Pakistan. There are a number of health hazards associated with obesity, including diabetes, hypertension, cardiovascular disease, arthritis, anesthesia risk, respiratory problems, breast cancer, menstrual abnormalities, ovarian dysfunction along with poor social image and rejection.

\section{Measure of Obesity}

Obesity can be assessed in several ways. Each method has advantages and disadvantages, and the appropriateness and scientific acceptability of each method will depend on the situation Body mass index (BMI). [9]

- Waist circumference

- Waist to hip ratio

\subsection{The BMI (Body Mass Index)}

BMI which describes relative weight for height correlates with both morbidity and mortality. The relative risk for diabetes and CVD (cardio vascular diseases) incidence increase in a graded fashion with increasing BMI in all population groups. BMI is significantly correlated with total body fat content. Moreover, calculating BMI is simple, rapid, and inexpensive, and can be applied generally to adults. Body mass index (BMI) has traditionally been used to identify individuals who are the most likely to be overweight 
or obese. It is calculated by dividing the weight (in kilograms) by the height (in meters) squared. Generally, a high value indicates excessive body fat and consistently relates to increased health risks and mortality [10].

\subsection{Waist Circumference}

Fat located in the abdominal region is associated with greater health risks than that in peripheral regions. Nonetheless, the presence of increased total abdominal fat appears to be an independent risk predictor when BMI is not markedly increased. Therefore, waist or abdominal circumference, as well as BMI, should be measured for the initial assessment of obesity.

\subsection{Waist to Hip Ratio}

Waist-to-hip ratio (WHR) is the ratio of the circumference of the waist to that of the hips. It is calculated by measuring the hip circumference at its widest part and dividing that into the waist circumference (located just above the upper hip bone). WHR is 0.7 for women and 0.9 for men have been shown to correlate strongly with general health and fertility. Women within the 0.7 and men with 0.9 ranges are less susceptible to major diseases such as diabetes, and cardiovascular disorders. WHR is a better predictor of mortality in older people than waist circumference or BMI. If obesity is redefined using WHR instead of BMI, the proportion of people categorized as at risk of heart attack worldwide increases threefold.

\section{Diabetes and Obesity}

A worldwide epidemic exists with respect to diabetes mellitus, primarily because of increased rates of obesity. Obesity has become widespread in developed countries along with a corresponding increase in the prevalence of diabetes mellitus. Epidemiological studies have shown that, compared to lean individuals, very obese men and women (body mass index $>35$ ) have a 60 - and 90 -fold increased probability of developing diabetes, respectively. Although the precise underlying mechanisms in the development of diabetes are as yet unknown, the initial patho-physiological event is usually insulin resistance, which involves a genetic component that is exacerbated by obesity and a sedentary lifestyle. There is a significant correlation between obesity and insulin resistance in nondiabetic subjects, and obesity exacerbates insulin resistance in diabetic subjects. However, the degree of insulin resistance that accompanies obesity varies considerably, and the relation among obesity, insulin resistance, and diabetes mellitus is not well understood [11].

The pancreas secretes insulin in order to help metabolize glucose and carbohydrates. When a non-diabetic person consumes excessive calories and gains weight, tissues become insensitive to insulin, and the body becomes less able to convert glucose into energy and other metabolites. One study has shown a decline in insulin sensitivity (or effectiveness) of 30 to 40 percent when a person exceeds 35 to 40 percent above their ideal weight. The body compensates by producing more insulin, a condition called hyperinsulinemia. Many studies have shown that obese adults have much higher insulin levels in their blood than leaner people. Increased adipose tissue especially that in an upper body or "android" deposition was first associated with diabetes and vascular disease by French endocrinologist Jean Vague in 1956. Insulin resistance increases with increasing body mass index, waist circumference and in particular waist-hip ratio [12]...

Table 1. Adiposites Secreted Proteins

\begin{tabular}{|c|c|c|c|}
\hline $\begin{array}{c}\text { Serial } \\
\text { no. }\end{array}$ & Category of protein & Name of protein & Expression in diabetics \\
\hline \multirow{2}{*}{1} & $\begin{array}{c}\text { Cytokines and cytokine- related } \\
\text { proteins }\end{array}$ & Leptin & + \\
& TNF- alpha & + \\
\hline 2 & IL-6 & + \\
\hline \multirow{2}{*}{3} & Proteins involved in fibrinolytic & MCP-1 & + \\
\hline \multirow{4}{*}{4} & system & PAI-1 & + \\
& Complement and complement- & Tissue factor & + \\
\hline \multirow{4}{*}{5} & related proteins & Adipsin (complement factor D) & + \\
& & ASP & + \\
& & Adiponectin & - \\
& & Lipoprotein lipase (LPL) & - \\
& & Apolipoprotein E & + \\
& Lipids and proteins for lipid & Apolipoprotein A1, & - \\
& metabolism or transport & Apolipoprotein A2 & - \\
& & Apolipoprotein B & + \\
& & Apolipoprotein H & + \\
\hline
\end{tabular}




\section{Proteins Secreted by Adiposites in Obese Condation}

Recent studies have established adipose tissue as an endocrine organ capable of hormone and cytokine secretion. Knowledge about this metabolic syndrome has moved forward with the description of numerous adipocyte secretary products. Adipocytes express and secrete several adipokines that are similar in their function to endocrine hormones such as leptin and adiponectin. Many secreted proteins are derived from the nonadipocyte fraction of adipose tissues.) [13] Knowledge of adipocyte biology is therefore crucial for understanding the pathophysiological basis of obesity and metabolic diseases such as diabetes mellitus as show in Table 1

\section{Adiposite Secreted Proteins with Role in Diabetes}

\subsection{Proteins Up Regulated in Obesity and Diabetes}

\subsubsection{Leptin}

Leptin, a 16000 dalton protein was the first adipokine discovered to have a role in modulating adiposity, and it remains the best studied. This obesity-related hormone is a molecule critical to the regulation of energy balance and body weight. Like adiponectin, it is secreted mainly by adipocytes. However, unlike adiponectin, which is inversely proportional to body fat, leptin levels have a direct correlation with total body fat and with increased serum levels in those with diabetes mellitus. Leptin also improves glucose homeostasis in lipodystrophic mice, and in humans with lipodystrophy or congenital leptin deficiency.

\subsubsection{C reactive Protein}

C-reactive protein (CRP) is an acute-phase protein which can have both pro- and anti-inflammatory effects.CRP is a member of the pentarxin family of proteins and is composed of five identical subunits, non-covalently linked to form a symmetrical disc of approximately 115000 Dalton. First identified in 1930, C-reactive protein is a nonglycosylated protein. C-reactive protein plays a role in destroying infectious agents, minimizing tissue damage, and facilitating tissue repair and regeneration. The concentration of CRP in the blood of healthy human beings ranges from 0 to $1.0 \mathrm{mg} / \mathrm{dL}$, but during acute inflammation, the CRP levels may increase 1000-fold. In humans, CRP level is an important indicator for the early, rapid diagnosis of a disease, especially for acute typhlitis, cholecystitis, and pancreatitis. In addition the C-reactive protein is also used as a novel predictor of cardiovascular events and of progression to diabetes mellitus in pre-diabetic individuals [14].

\subsubsection{Apolipoprotein B}

Apolipoprotein B (apoB) is the primary apolipoprotein of low density lipoproteins (LDL) which are called bad cholesterol and are responsible for carrying cholesterol to tissues. This 250000 dalton protein is the primary apolipoprotein component of LDL and is absolutely required for its formation. What is clear is that the apoB on the LDL particle acts as a ligand for LDL receptors in various cells throughout the body. Through a mechanism that is not fully understood, high levels of apoB can lead to plaques that cause heart disease (atherosclerosis). High plasma levels of apoB are positively corelated to serum LDL cholesterol level and thus are risk factors for atherosclerosis, a leading cause of death in the population and important marker to check the progression of obesity related diabetes mellitus.

\subsubsection{Apolipoprotein E}

Apolipoprotein E (apoE) is essential for the normal catabolism of triglyceride-rich lipoprotein constituents. ApoE was initially recognized for its importance in lipoprotein metabolism and cardiovascular disease. ApoE is 34000 dalton protein and transports lipoproteins, fat-soluble vitamins, and cholesterol into the lymph system and then into the blood. However, increased expression of apoE increases the rate of hepatic VLDL (very-low-density lipoprotein), triacylglycerol secretion and is associated with hypertriglyceridaemia. In humans and experimental animals, plasma apoE levels are correlated with plasma triacylglycerol levels [15].

\subsubsection{Alpha 2 Macroglobulin}

Alpha 2-macroglobulin ( $\alpha 2-\mathrm{M})$, a proteinase inhibitor in blood and tissue, is known to act as such a binding protein for numerous cytokines and growth factors. Recently, we have shown that $\alpha 2-\mathrm{M}$ is also involved in binding and transport of human growth hormone. These biologically important polypeptides mainly bind to transformed $\alpha 2-\mathrm{M}$ (a2-Mt), which is the receptor recognizable form of the inhibitor. The receptor of $\alpha 2-\mathrm{M}(\alpha 2-\mathrm{M}-\mathrm{R})$ was found to be identical to the low density lipoprotein receptor-related protein (LRP). The proteinase inhibitor, a2-M, may act as a leptin-binding protein in human plasma. Binding of leptin to transformed a2-M and its rapid clearance by the a2-M receptor may significantly influence the bioavailability of leptin in human plasma [16].

\subsubsection{Lipoprotein Lipase}

Lipoprotein lipase is a key enzyme in lipid homeostasis in humans, providing intravascular release of fatty acid from circulating triacylglycerol. Lipoprotein lipase is the primary enzyme that converts lipoprotein triglyceride (TG) to FFAs (free fatty acids). A genetic deficiency of LPL results in hyperlipoproteinemia. LPL is produced by heart, adipose tissue, muscle, as well as in small amounts by many other tissues. It is a glycoprotein anchored on the luminal surface of capillary endothelium through its interaction with cell surface glycosaminoglycans. The normal targets for LPL 
action are the triacylglycerol-rich lipoproteins, especially chylomicrons and very low density lipoproteins (VLDL). Abnormalities in LPL function have been found to be associated with a number of pathophysiological conditions, including atherosclerosis, chylomicronaemia, obesity, Alzheimer's disease, and dyslipidaemia associated with diabetes, insulin resistance, and infection [16].

\subsubsection{Tumor necrosis Factor Alpha}

Tumor necrosis factor $\alpha$ (TNF- $\alpha)$ is a pleiotropic cytokine that plays an important role in immunity and inflammation. During chronic illness, markedly elevated TNF- $\alpha$ secretion can contribute to hemorrhage, necrosis, and in severe cases, death. Overproduction of TNF- $\alpha$ is thought to play a role in a number of disease processes including arthritis, periodontal disease, inflammatory bowel disease, and chronic obstructive pulmonary disease. In each case, TNF- $\alpha$ is associated with persistent inflammation and tissue destruction. The inflammatory events stimulated by TNF- $\alpha$ can lead to connective tissue destruction by the release of lytic enzymes produced by resident cells as well as by recruited inflammatory cells. Diabetes is associated with excessive TNF- $\alpha$ expression. This may result from constitutive overproduction by adipose tissue in diabetes mellitus, the effects of hyperglycemia and advanced glycation end products and an exaggerated or more persistent response to stimuli such as bacteria or wound healing. TNF- $\alpha$ overexpression in diabetes is thought to contribute to several complications in diabetes, including retinopathy, nephropathy, neuropathy, and diabetes-enhanced periodontal disease. Delayed or incomplete healing of wounds has been well documented in diabetic humans and in animal models of diabetes [17] .

\subsection{Proteins Down Regulated in Obesity and Diabetes}

\subsubsection{Adiponectin}

One of the adipokines adiponectin, molecular weight 30000 dalton is a collagen-like plasma protein produced and secreted exclusively by adipose tissue, has been shown to have compelling anti-atherogenic, and insulin-sensitizing properties. Cross-sectional studies of human subjects have reported decreased concentrations of adiponectin in patients with diabetes mellitus, hypertension, dyslipidemia, and cardiovascular disease compared with healthy individuals, and weight reduction has resulted in increases in adiponectin. In addition, inverse correlations of adiponectin with BMI, percent body fat, waist-to-hip ratio, glucose, insulin, and triglyceride and positive correlations with HDL and direct measures of insulin sensitivity have been consistently demonstrated [18].

\subsubsection{Apolipoprotein A-I}

Apolipoprotein A1 (ApoA-I) is the major protein component of high density lipoprotein (HDL) in plasma. This protein, of molecular weight 28000 dalton, promotes cholesterol efflux from tissues to the liver for excretion. The level of apo A1 is positively corelated with HDL cholesterol in the serum and negitively corelated with LDL cholesterol. ApoA-I is synthesized in both the intestine and the liver, but the relative contribution of the intestine and the liver to the plasma apoA-I pool in humans remains unknown.

\subsubsection{Apolipoprotein AII}

Apolipoprotein AII (ApoA-II) constitutes approximately $20 \%$ of HDL protein, is present on about two-thirds of HDL particles in humans, and is synthesized only in the liver. ApoA-II is also required for normal HDL biosynthesis and metabolism. This protein just like apoA1 is present in low concentration in obese [19].

\subsubsection{Apolipoprotein $\mathrm{H}$}

Apolipoprotein $\mathrm{H}(\mathrm{ApoH})$, also known as $\beta 2$-glycoprotein $\mathrm{I}$, is a plasma glycoprotein either circulating as a free protein or associated to lipoproteins. ApoH is a 54000 dalton single-chain glycoprotein consisting of five carbohydrate chains. Reports show that ApoH may have an important function in blood coagulation and clearance of apoptotic bodies from the circulation. Some works indicate that the binding of ApoH with target membranes containing anionic phospholipids could induce agglutination and precipitation. The interactions of ApoH with phospholipids are considered crucial in explaining its physiological or clinical roles [20-21].

\section{Aims and Objective of the Study}

The study was carried out to check the effect of obesity on diabetes and how certain proteins which have their origin in the adipose tissues can cause the aggravation and progression of diabetes mellitus. In our study, firstly, we have focused on the effect of obesity on the lipid profile and other biochemical parameters such as protein concentration and fasting blood glucose level of the of diabetic individuals. Secondly we have checked the expression level of various proteins which are secreted by adipose tissues in the serum of diabe2ic obese subjects and the possible effect they have on glucose homeostasis or generally on the progression of diabetes mellitus.

\section{REFERENCES}

[1] American diabetes association 2004. Diagnosis and classification of diabetes mellitus, Diabetes Care. 27:S5-S10.

[2] Samreen Riaz, James Larkin, M Waheed Akhtar, Tahir Shafi and Paul J Thornalley. 2009. High dose thiamine therapy for people with type 2 diabetes and microalbuminuria: a randomised, double-blind, placebo-controlled study. Diabetologia. Springer Berlin / Heidelberg. Vol. 52 (2), 208-212 
[3] Samreen Riaz, Mehreen Raza, Saadia Shhazad Alam, Shahida Hasnain and M. Waheed Akhtar. 2009. Obesity as risk factor and study of obesity related proteins in diabetes mellitus. African Journal of Biotechnology Vol. 8 (5), pp. 737-744, 6 March, 2009.

[4] Samreen Riaz, Riaz, S. and Hasnain, S. 2009. Screening of anti Methicillin Resistant Staphylococcus aureus (MRSA) bacteriocin Producing Bacteria. African Journal of Biotechnology. Vol. 8 (3), pp. 365-368. February Issue 2009.

[5] Samreen Riaz, Larkin, J.R., Akhtar, M.W., Shafi, T. and Thornalley, P.J. Response to comment on Rabbani et al., (2009) High dose thiamine therapy for patients with type 2 diabetes and microalbuminuria: a pilot randomised, double-blind, placebo-controlled study. Diabetologia, 52(2): 208 - 212, 2009. Diabetologia. 52 (6): 1214-1216.

[6] Samreen Riaz Diabetes: Could vitamin 'B' the answer? Chloë Harman Comments on Original article, Nature Reviews Endocrinology 5(5), 236-236. Research Highlights

[7] Samreen Riaz Diabetes: Could vitamin 'B' the answer? Chloë Harman Comments on Original article, Naila Rabbani, Saadia Shahzad Alam, Samreen Riaz, James Larkin, M Waheed Akhtar, Tahir Shafi and Paul J Thornalley. High dose thiamine therapy for people with type 2 diabetes and microalbuminuria: a randomised, double-blind, placebo-controlled study. Diabetologia 52, 208-212 (2009). Nature Reviews Nephrology 5 (4), 182.

[8] Samreen Riaz, Diabetes mellitus (Review article) Scientific Research and Essay. Vol. 4 (5) pp. 367-373, May, 2009.

[9] Samreen Riaz, Saadia Shahzad Alam and M. Waheed Akhtar. (2010). Proteomic Identification of human serum biomarkers in diabetes mellitus type-2. Journal of Pharmaceutical and Biomedical Analysis. Volume 51 issue 5, 1103-1107.

[10] Samreen Riaz and Ansa Butt. Study of Protein profiling of human urine in diabetic hypertensive nephropathy versus normal healthy controls. Diabetes technology \& Therapeutics. 2010. 12 (5): 379-386

[11] Samreen Riaz, Saadia Shahzad Alam, Surjit Kaila Srai, Vernon Skinner, Aasma Riaz and M. Waheed Akhtar. (2010). Proteomic Identification of human urine biomarkers in diabetes mellitus type-2. Journal of Diabetes technology \& Therapeutics. 2010. 12 (12): 979- 988.

[12] Samreen Riaz, Vernon Skinner and Surjit Kaila Srai. (2011). Effect of high dose thiamine on levels of human urine protein biomarkers in diabetes mellitus type 2. Journal of Pharmaceutical and Biomedical Analysis. 201154 (4):
$817-825$.

[13] Samreen Riaz, Vernon Skinner and Surjit Kaila Srai. (2011). Corrigendum to "Effect of high dose thiamine on the levels of urinary protein biomarkers in diabetes mellitus type 2" [J. Pharm. biomed. Anal. 54 (2011) 817-825], Journal of Pharmaceutical and Biomedical Analysis. 2011. 56 (1): 139.

[14] Saadia Shahzad Alam, Samreen Riaz and M. Waheed Akhtar. (2012) Effect of high dose thiamine therapy on activity and molecular aspects of transketolase in Type 2 diabetic patients. African Journal of Biotechnology Vol. 10(75), pp. 17305-17316.

[15] Saadia Shahzad Alam, Samreen Riaz and M. Waheed Akhtar. (2012). Effect of High Dose Thiamine Therapy on Risk Factors in Type 2 Diabetics. J Diabetes Metab (IF 3.784) 2012, 3:10,

[16] Saadia Shahzad Alam, Samreen Riaz and M. Waheed Akhtar. (2013) Effect of high dose thiamine therapy on induction and activities of pyruvate dehydrogenase and $\alpha$-ketoglutarate dehydrogenase in type 2 diabetic patients. Diabetes Medicine. Manuscript in progress.

[17] Samreen Riaz, "Study of Protein Biomarkers for Diabetes Mellitus Type 2 and Role of High Dose Thiamine on their Level" Advances in Medicine and Biology (2010). INova publishs\Advances in Medicine and Biology_Volume 13.htm.Chapter 11. Volume 13. page numbers. 163-176

[18] Samreen Riaz and Saadia Shahzad Alam (2012-14), STUDY OF DIABETIC HYPERTENSIVE NEPHROPATHY IN THE LOCAL POPULATION OF PAKISTAN. Diabetic Nephropathy, Laboratory of Molecular Nephrology and Endocrinology, CRCHUM- Hôtel-Dieu Hôpital Montréal, Québec, Canada. ISBN 978-953-308- p147-166.Treatement of diabetes type 2. Intech Publsihers.

[19] Samreen Riaz. Protein Biomarkers for diabetes mellitus type 2. Biomarker. (2012). Book edited by: Assoc. Prof. Tapan Kumar Khan, Blanchette Rockefeller Neurosciences Institute at West Virginia University, USA. ISBN 979-953-307-612-5.

[20] Diabetes Prevention Program Research Group, 2005. Diabetes Care. 28(10): 2472-2479.

[21] The Expert Committee on the Diagnosis and Classification of Diabetes Mellitus, Report of the Expert Committee on the Diagnosis and Classification of Diabetes Mellitus, 1997. Diabetes Care 20: 1183-1197.

[22] The Expert Committee on the Diagnosis and Classification of Diabetes Mellitus: Follow-up report on the diagnosis of diabetes mellitus, 2003. Diabetes Care 26: 3160-3167. 